\title{
Regulationsverhalten von Auto-CPAP-Geräten während der Simulation von schlafbezogenen Atemflussmustern
}

\section{Characteristics of Auto-CPAP Devices During the Simulation of Sleep-Related Breathing Flow Patterns}

Autoren

Institute
K. H. Rühle ${ }^{1}$, D. Karweina ${ }^{2}$, U. Domanski ${ }^{1}$, G. Nilius ${ }^{1}$

Klinik für Pneumologie, HELIOS-Klinik Ambrock Hagen, Universität Witten-Herdecke

Fachbereich Elektrotechnik und Informationstechnik, Fachhochschule Südwestfalen eingereicht 14.1.2009

akzeptiert nach Revision 27. 4. 2009

Bibliografie

DOI $10.1055 / \mathrm{s}-0029-1214746$

Pneumologie 2009; 63:

390-398 @ Georg Thieme

Verlag KG Stuttgart · New York ISSN 0934-8387

Korrespondenzadresse Prof. Dr. med. Karl-Heinz Rühle HELIOS-Klinik Ambrock Klinik für Pneumologie 58091 Hagen

Klinik-Ambrock.Pneumo@ t-online.de

\section{Zusammenfassung \\ $\nabla$}

Einleitung: Die Funktionsweise automatischer CPAP-Geräte (APAP) ist mit klinischen Untersuchungen aufgrund der hohen Variabilität der Atmungsstörung nur schlecht zu untersuchen. Mit einem Flussgenerator können identische Atemmuster reproduziert werden, sodass vergleichende Untersuchungen zum Druckverhalten von APAP-Geräten möglich werden. Da die Algorithmen von APAP-Geräten aufgrund der Erfahrungen der Anwender ohne größeren Aufwand durch den Hersteller modifiziert werden können, sollten auch bereits auf dem Markt eingeführte Geräte regelmäßig auf Programmveränderungen überprüft werden.

Fragestellung: Wurden im Vergleich zu den bisher publizierten Bench-Studien Veränderungen der Steuerprogramme von 3 ausgewählten Geräten durchgeführt und stimmen unsere Ergebnisse mit den vorangegangenen Untersuchungen überein? Differenzieren die aktuell untersuchten Geräteversionen zwischen offenen und geschlossenen Apnoen?

Methodik: Mit einer selbstentwickelten reglergesteuerten Atempumpe wurden schlafbezogene Atemflussmuster sowie mit einem rechnergesteuerten Ventil die Widerstände der oberen Atemwege simuliert. Drei verschiedene AutoCPAP-Geräte wurden einem Bench-Test ohne und mit Rückkopplung (open/closed loop) unterzogen.

Ergebnisse: Open loop: Die Geräte zeigten deutliche Unterschiede in der Geschwindigkeit des Druckanstiegs, unterscheiden sich aber nicht von den früheren publizierten Ergebnissen. Bei einem Ausgangsdruck von jeweils 4 mbar erfolgte der Druckanstieg bis 10 mbar nach einer unterschiedlichen Anzahl von Apnoen (1-6 repetitive Apno-

\section{Abstract \\ $\nabla$}

Introduction: The function of automatic CPAP devices is difficult to investigate using clinical examinations due to the high variability of breathing disorders. With a flow generator, however, identical breathing patterns can be reproduced so that comparative studies on the behaviour of pressure of APAP devices are possible. Because the algorithms of APAP devices based on the experience of users can be modified without much effort, also previously investigated devices should regularly be reviewed with regard to programme changes.

Questions: Had changes occurred in the algorithms of 3 selected devices - compared to the previously published benchmark studies? Do the current versions of these investigated devices differentiate between open and closed apnoeas?

Method: With a self-developed respiratory pump, sleep-related breathing patterns and, with the help of a computerised valve, resistances of the upper respiratory tract were simulated. Three different auto-CPAP devices were subjected to a bench test with and without feedback (open/closed loop).

Results: Open loop: the 3 devices showed marked differences in the rate of pressure rise but did not differ from the earlier published results. From an initial pressure of 4 mbar the pressure increased to 10 mbar after a different number of apnoeas ( $1-6$ repetitive apnoeas). Only one device differentiated between closed and open apnoeas. Closed loop: due to the pressure increase, the flow generator simulated reduced obstruction of the upper airways (apnoeas changed to hypopnoeas, hypopnoeas changed to flattening) but different patterns of pressure regulation could still be observed. 
en). Nur ein Gerät differenzierte zwischen geschlossenen und offenen Apnoen. Closed loop: Die durch die Druckerhöhung reduzierte Obstruktion der oberen Luftwege (aus Apnoe wird Hypopnoe, aus Hypopnoe wird Flattening) führte in den 3 Geräten zu unterschiedlichen Mustern der Druckregulation.

Schlussfolgerung: Durch Bench-Tests kann der Algorithmus der APAP-Geräte regelmäßig überprüft und Veränderungen der Software erkannt werden. Die Differenzierung zwischen offenen und geschlossenen Apnoen sollte bei einigen APAP-Geräten verbessert werden.
Conclusion: By applying bench-testing, the algorithms of autoCPAP devices can regularly be reviewed to detect changes in the software. The differentiation between open and closed apnoeas should be improved in several APAP devices.

\section{Einleitung und Fragestellung}

Um die Reaktions-Charakteristika automatischer CPAP-Geräte (APAP) abschätzen zu können, sollten unter definierten Bedingungen identische Atemmuster reproduzierbar für Vergleichsuntersuchungen (sogenannte Bench-Tests) zur Verfügung stehen.

Dies ist erforderlich, da die Auto-CPAP-Geräte unterschiedliche Parameter wie zum Beispiel Atemfluss, Flattening, Widerstandmessungen mit der forcierten Oszillations-Technik (FOT) oder Schnarchen zur Steuerung des Druckverhaltens verwenden. Die Signale werden anhand der in den Geräten integrierten Software mittels verschiedener Algorithmen bewertet, sodass die Druckvariabilität unterschiedlich ausfällt [1].

Bis vor wenigen Jahren war es nicht möglich, das Verhalten von APAP-Geräten zu testen, da geeignete Flussgeneratoren, die die typischen Flussmuster bei schlafbezogenen Atemstörungen (SBAS) erzeugen, nicht zur Verfügung standen. Eine spanische Arbeitsgruppe stellte als erste ein Mess-Protokoll vor, in dem anhand definierter, realistischer Flussmuster APAP-Geräte verglichen wurden [2]. Die Reaktion der getesteten APAP-Geräte zeigte beträchtliche Unterschiede in der Druckanstiegsgeschwindigkeit, nur eines von 5 überprüften Geräten reagierte auf eindeutige Hypopnoen (Amplitude < 50\%). Bei diesen Testungen wurde in regelmässigen zeitlichen Abständen eine Serie von Atemstörungen simuliert und der Druckanstieg des APAP-Gerätes registriert . Der Simulator reagiert also nicht auf den Druckanstieg. Es handelt sich um ein nicht rückgekoppeltes Verhalten, damit um ein Open-Loop-System.

Dies entspricht allerdings nicht der Situation am Menschen, da in der realen klinischen Situation durch die Druckerhöhung des APAP-Gerätes die oberen Atemwege erweitert und die Atemstörung vermindert oder beseitigt wird. Bei diesem Verhalten spricht man von einem rückgekoppelten oder Closed-Loop-System.

In einer weiteren Bench-Studie wurden die oberen Atemwege durch einen kollabilen Schlauch in einer Kammer mit variablem Umgebungsdruck - einem sogenannten Starling-Resistor - und die Atmung mit einer Pumpe, die sinusoidale Flüsse erzeugte, simuliert. Damit konnte die Reaktion der APAP-Geräte in einem rückgekoppelten System (closed loop) beobachtet werden [3]. In einer weiteren Studie wurden mit einer 2-Kammer-MichiganTestlunge verschiedene abgeflachte inspiratorische Flusskurven erzeugt, um die Sensitivität der Geräte für verminderte Atemflüsse zu überprüfen [4]. Schließlich wurde ein Simulationsmodell entwickelt, mit dem die Reaktion auf obstruktive und zentrale Apnoen/Hypopnoen untersucht werden konnte. Durch Einfügen eines servogesteuerten Ventils wurden die jeweiligen Widerstände der oberen Atemwege synchron zu dem vom Flussgenerator erzeugten Atemfluss simuliert. Im Folgenden wird von geschlossenen und offenen Apnoen/Hypopnoen gesprochen.
Außerdem wurde eine Closed-Loop-Simulation durchgeführt, wobei die Reaktion der Atemwege auf die Druckerhöhung durch APAP berücksichtigt wurde [5].

Da sich die Software der APAP-Geräte sehr schnell ändern lässt, können die Algorithmen durch die Gerätehersteller leicht umprogrammiert werden. Diese evtl. veränderten Reaktionsmuster sollten durch regelmäßige Bench-Studien erfasst werden. Zur Durchführung einer Vergleichstudie entwickelten wir deshalb einen reglergesteuerten Flussgenerator.

Die Gleichwertigkeit der getesteten APAP-Geräte mit konstantCPAP bei gleichzeitiger Senkung des nächtlichen mittleren Drucks wurde bereits in vielen Studien nachgewiesen [6-9]. Aus grundsätzlichen Überlegungen zur Pathophysiologie der obstruktiven Schlaf-Apnoe lässt sich ableiten, dass möglichst sämtliche obstruktive Atmungsstörungen in der Nacht vermieden werden sollten. Jedoch ist ein optimaler Therapie-Algorithmus nicht bekannt, da einerseits möglichst alle Atmungsstörungen vermieden werden sollten, andererseits zu schnelle Änderungen des Drucks zu Weckreaktionen und einer Verminderung der Schlafqualität führen könnten. Daher kann die klinische Wertigkeit der Geräte durch einen solchen Bench-Test nicht beurteilt werden.

Wir fragten uns:

- Stimmen unsere Ergebnisse mit den vorangegangenen BenchStudien überein?

- Wurden im Vergleich zu den bis 2006 publizierten Studien Veränderungen der Algorithmen durchgeführt? Insbesondere interessierte, ob neuere Geräteversionen zwischen offenen und geschlossenen Apnoen differenzieren können.

Verglichen werden sollten Auto-CPAP-Geräte von drei verschiedenen Herstellern, wobei ein Gerät in der Untersuchung von Rigau et al. [5] bereits obstruktive von nicht obstruktiven Ereignissen unterscheiden konnte.

\section{Methodik}

$\nabla$

Reglergesteuerter Flussgenerator (Atempumpe): Zur Simulation der Pumpbewegung dient eine Kolbenpumpe (Zylinder-Innendurchmesser $100 \mathrm{~mm}$, Zylinderlänge $950 \mathrm{~mm}$ ), die über einen Linearantrieb und dem dazugehörigen Gleichstrommotor angetrieben wird (siehe Abb.1). Damit kann eine Totalkapazität von bis zu 7 Litern simuliert werden. Die Ansteuerung des Motors erfolgt über einen Mikrocontroller. Ausgehend von den physiologischen Zusammenhängen (siehe $\bullet$ Abb.2) der natürlichen Atmung wurde durch die Entwicklung einer geeigneten Regelstruktur versucht, eine möglichst gute Übereinstimmung in den wesentlichen physikalischen Größen zwischen der natürlichen Atmung und dem Atmungs-Simulator (siehe Abb. 3) zu erzielen. Anhand der Messungen von Ösophagus-Druck (entspricht 


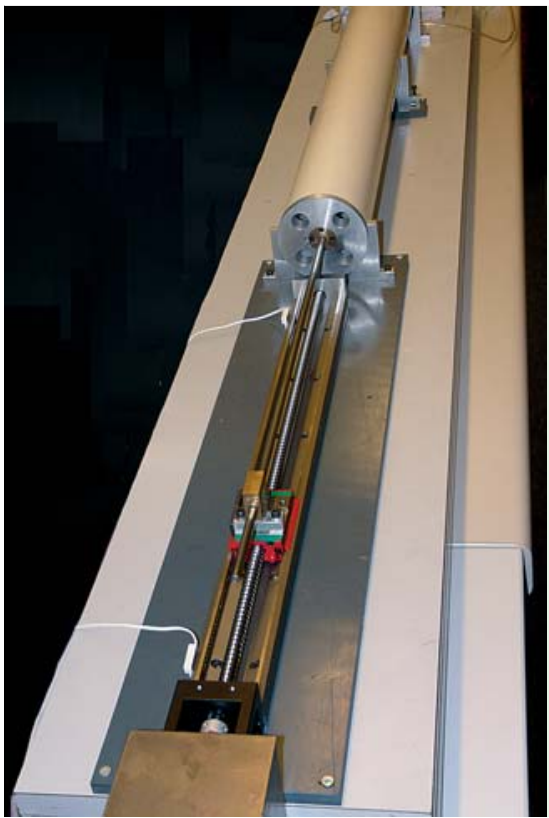

Abb. 1 Atempumpe.

dem Pleura-Druck) und Flow bei verschiedenen Patienten konnte eine gute Übereinstimmung zwischen der Atemdynamik des Patienten und der des Atmungs-Simulators nachgewiesen werden (Beispiel $\odot$ Abb. 4 ). Die aus aktuellen Messungen bei OSAS-Patienten ermittelten normalen bzw. bei Apnoe oder Hypopnoen auftretenden Flussprofile lassen sich in geeignete Steuersignale für den Atmungssimulator umrechnen, sodass diese Flussprofile nun repetitiv per Programm erzeugt werden konnten. Hochfrequente Vibrationen, wie sie beim Schnarchen entstehen, wurden nicht erzeugt.

\section{Widerstand der oberen Atemwege}

$\nabla$

Zur Simulation des Widerstandes der oberen Atemwege wurde ein spezielles Ventil entwickelt, bei dem einerseits der Querschnitt pneumatisch gesteuert verändert werden kann (siehe Abb. 5 u. 6) und das andererseits ein gewisses kollabiles Verhalten aufweist. Ein kollabiler Gummischlauch, durch den die Atemluft geführt wird, befindet sich in einem abgeschlossenen zylinderischen Raum, der mit Luft unterschiedlichen Drucks gefüllt werden kann. Das kollabile Element liegt zwischen der Pumpe und dem Atmungsschlauch, der zur Maske führt. Eine Druckregelung sorgt für die Einhaltung des gewünschten Drucks. Da bei diesem Ventil nur sehr geringe Massen bewegt werden müssen, kann die Querschnittsveränderung sehr schnell erfolgen, sodass auch Widerstandsveränderungen während eines Atem-Zyklus erfolgen können.

Mit dieser Anordnung (siehe Abb. 7) war es möglich, schlafbezogene Atemflussmuster mit und ohne Obstruktion zu simulieren. Die Messung von Fluss und Druck erfolgte hinter dem Obstruktionskörper. Über eine A/D-Wandlerkarte wurden die Daten mit einer Registrier-Frequenz von $1000 \mathrm{~Hz}$ auf einem digitalen Rechner aufgezeichnet und dort gespeichert.

Drei verschiedene Auto-CPAP-Geräte wurden einem Bench-Test ohne und mit Rückkopplung (open/closed loop) unterzogen. Dazu wurde der generierte Flow zu dem Kopf eines Dummy mit fixierter Mund-Nasenmaske geführt. Diese wurde mit einem Schlauch mit dem APAP-Gerät verbunden.
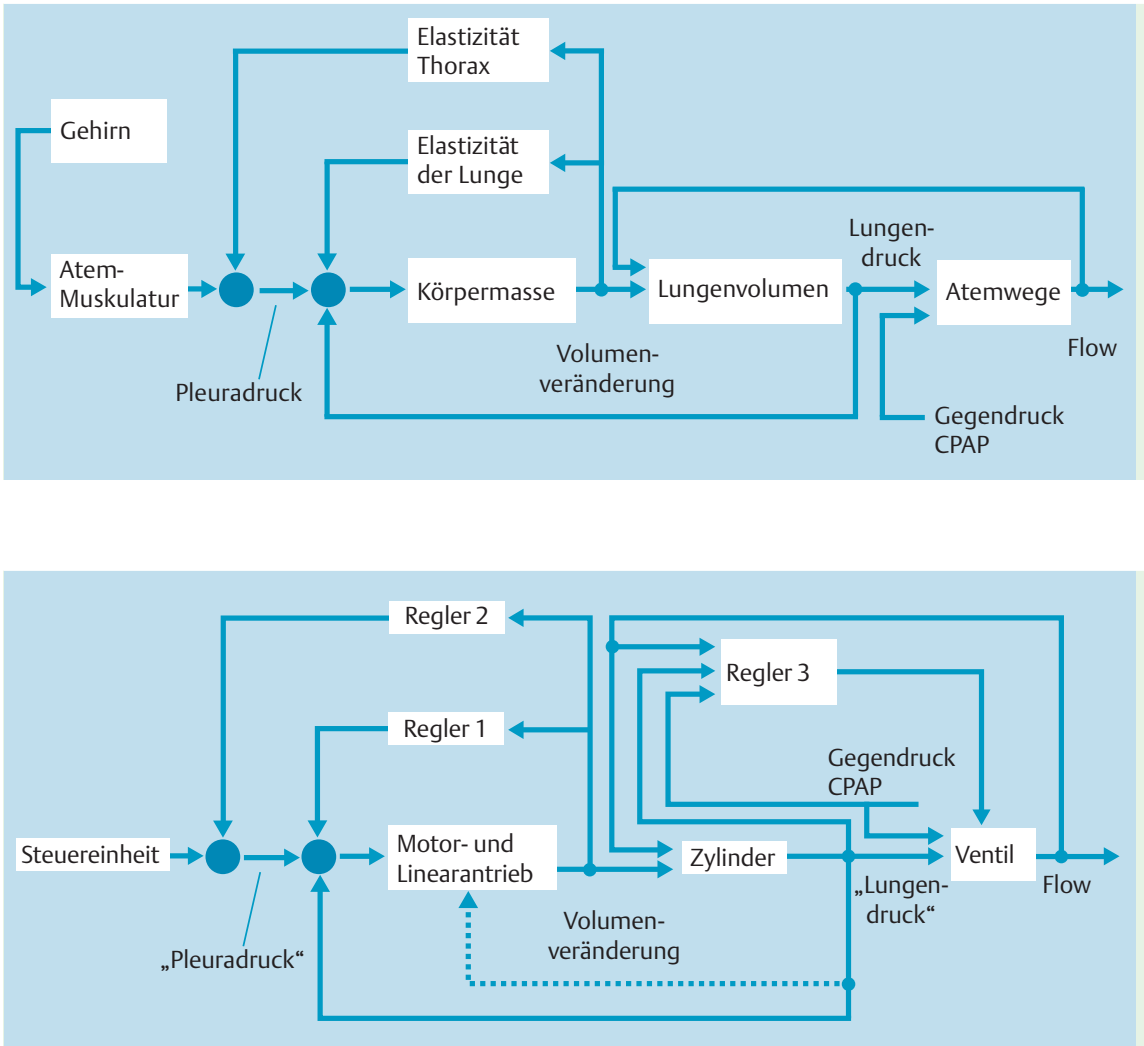

Abb. 2 Wirkungszusammenhänge bei der natürlichen Atmung.

Abb. 3 Wirkungszusammenhänge im AtmungsSimulator. 


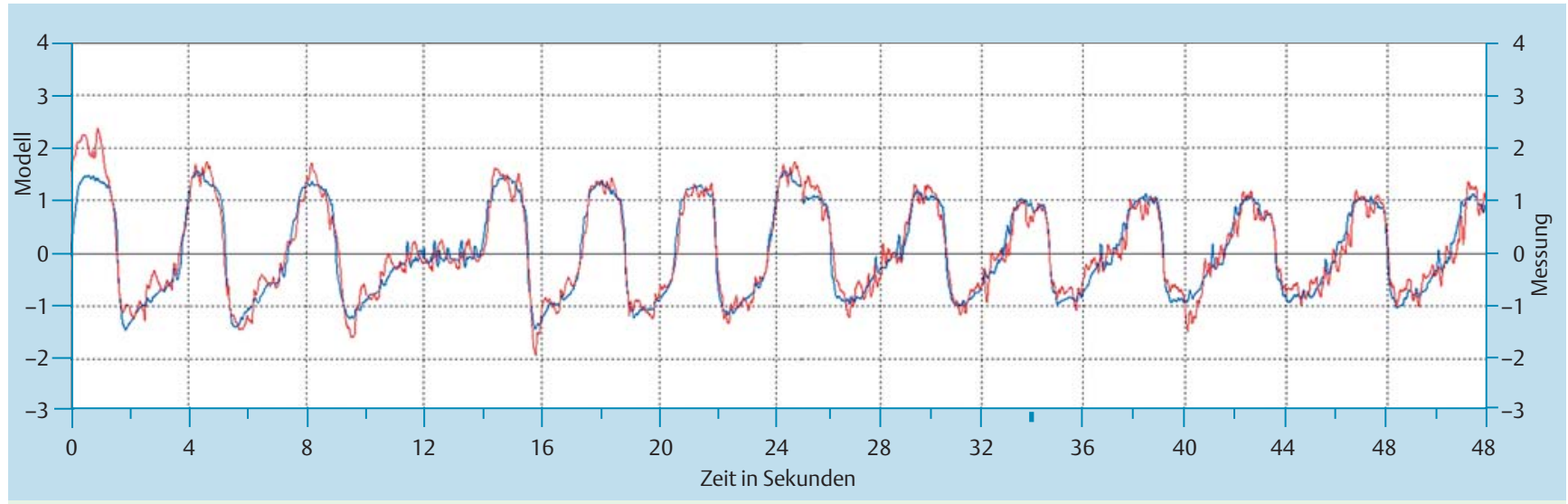

Abb. 4 Vergleich Originalregistrierung und Simulation.

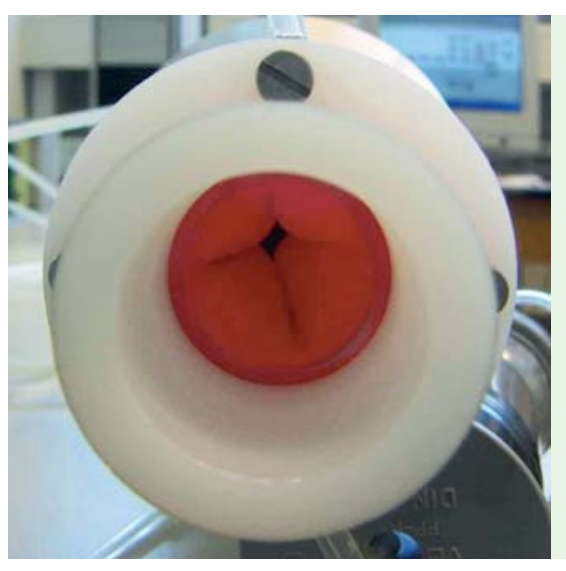

Abb. 5 Pneumatisch gesteuerter Verschlusskörper während der Simulation einer pharyngealen Obstruktion.

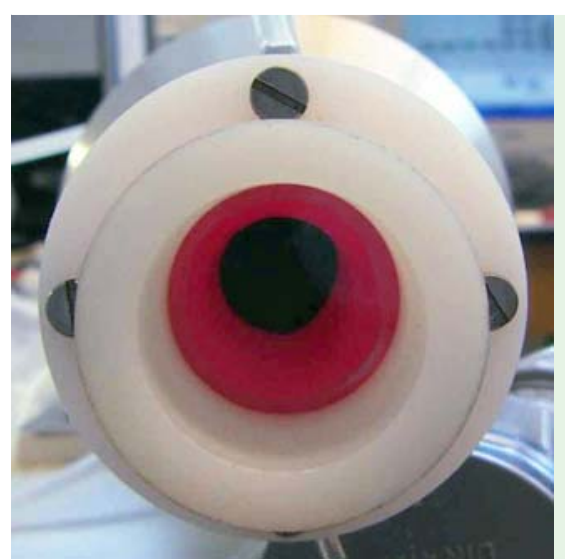

Abb. 6 Pneumatisch gesteuerter Verschlusskörper im geöffneten Zustand.

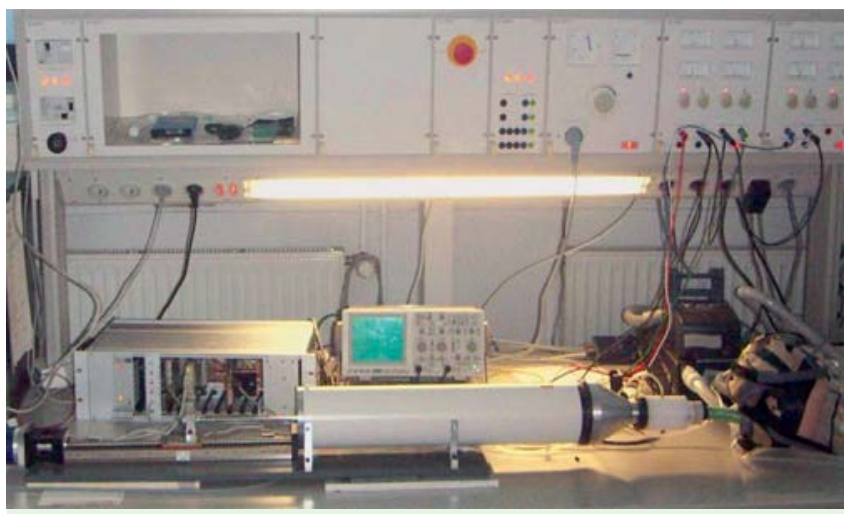

Abb. 7 Versuchsanordnung zur Untersuchung von APAP-Geräten. Von links: Gleichstrommotor, Linearantrieb, Kolbenpumpe, Verschlusskörper, Dummy mit Maske. Im Hintergrund das über einen Schlauch mit der Maske verbundene APAP-Gerät.

\section{Ablauf der Studie \\ $\nabla$}

Jeweils ein handelsübliches Gerät aus der laufenden Serie (April Juli 2007) wurde zur Testung ausgewählt. Getestet wurden Gerät A (S8, Resmed), Gerät B (Phönix, Respironics) und Gerät C (Somnosmart, Weinmann).

\section{Testlauf 1}

Obstruktive Apnoen: Die Registrierung von Druck und Fluss erfolgte beginnend bei Normalatmung bei $4 \mathrm{mbar}$. Die Messungen wurden zweimal durchgeführt und jeweils nach maximal $30 \mathrm{~min}$ beendet. Die Druck- und Flussdaten konnten mit einer Abweichung $<5 \%$ reproduziert werden. Zu Beginn von jeder Apnoe wurde automatisch die Öffnung des Ventilkörpers verschlossen und am Ende geöffnet.

\section{Testlauf 2}

Offene Apnoen: Die Untersuchung verlief genau wie in Testlauf 1, der Ventilkörper zu Beginn einer Apnoe wurde nicht verschlossen.

\section{Testlauf 3}

Closed loop: Die Programmierung des Flussgenerators wurde folgendermaßen geändert: Der Flusssimulator generierte bis 6 mbar Apnoen $($ Flow =0). Sobald der Flowgenerator des APAPGerätes reagierte und der CPAP den Wert von 6 mbar überschritt, wurden durch das Rechnerprogramm des Simulators die Apnoen in Hypopnoen (Flowreduktion um 50\%) umgewandelt. Bei weiterem Druckanstieg ab 7,5 mbar wurde Flattening und über 9 mbar Normalatmung simuliert.

\section{Ergebnisse \\ $\nabla$}

Open loop, obstruktive Apnoen: Alle 3 Geräte erhöhen am Ende einer Apnoe nach einer vorgegebenen Anzahl von Apnoen den Druck um einen bestimmten Betrag.

Gerät A: Bei einem Ausgangsdruck von 4 mbar erfolgte nach der ersten Apnoe ein Druckanstieg mit 2 mbar, danach mit 1 mbar und anschließend mit 0,5 mbar, um sich mit immer kleineren 


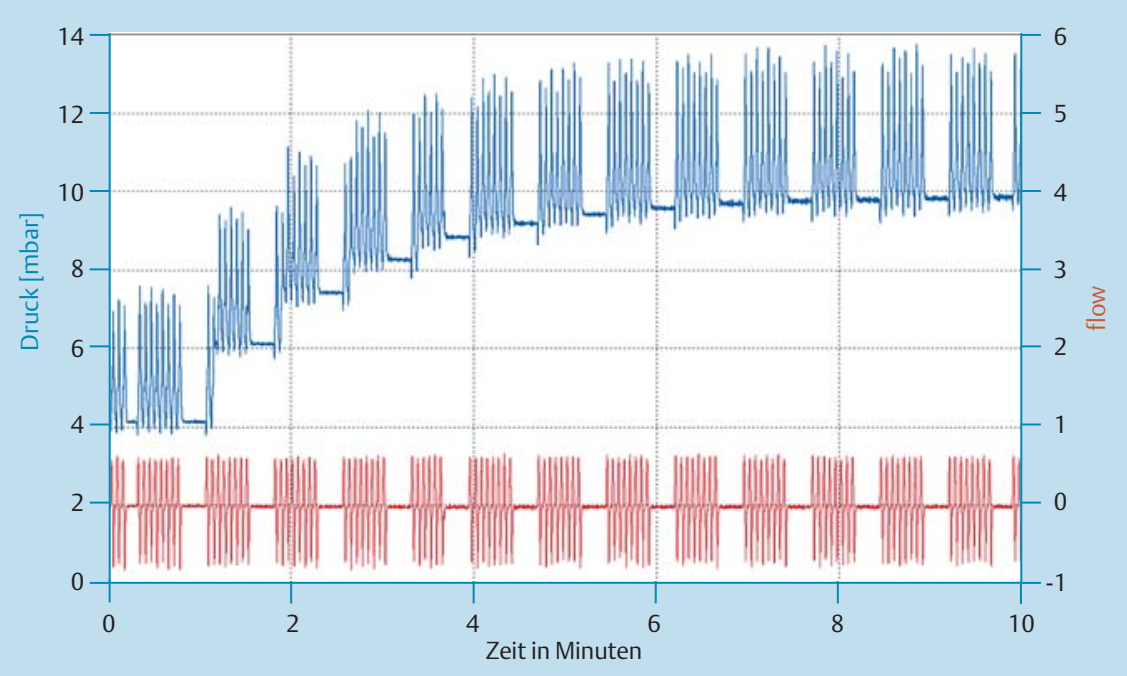

Abb. 8 Open-Loop-Druck (oben) und -Fluss (unten) während repetitiver obstruktiver Apnoen. Erklärung open/closed loop. Registrierzeit $10 \mathrm{~min}$ Verhalten von Gerät A: Ausgangsdruck 4 mbar. Nach erster Apnoe Druckanstieg 2 mbar, danach $1 \mathrm{mbar}, 0,5 \mathrm{mbar}$, jeweils Halbierung der Druckschritte, obere Druckgrenze 10 mbar.

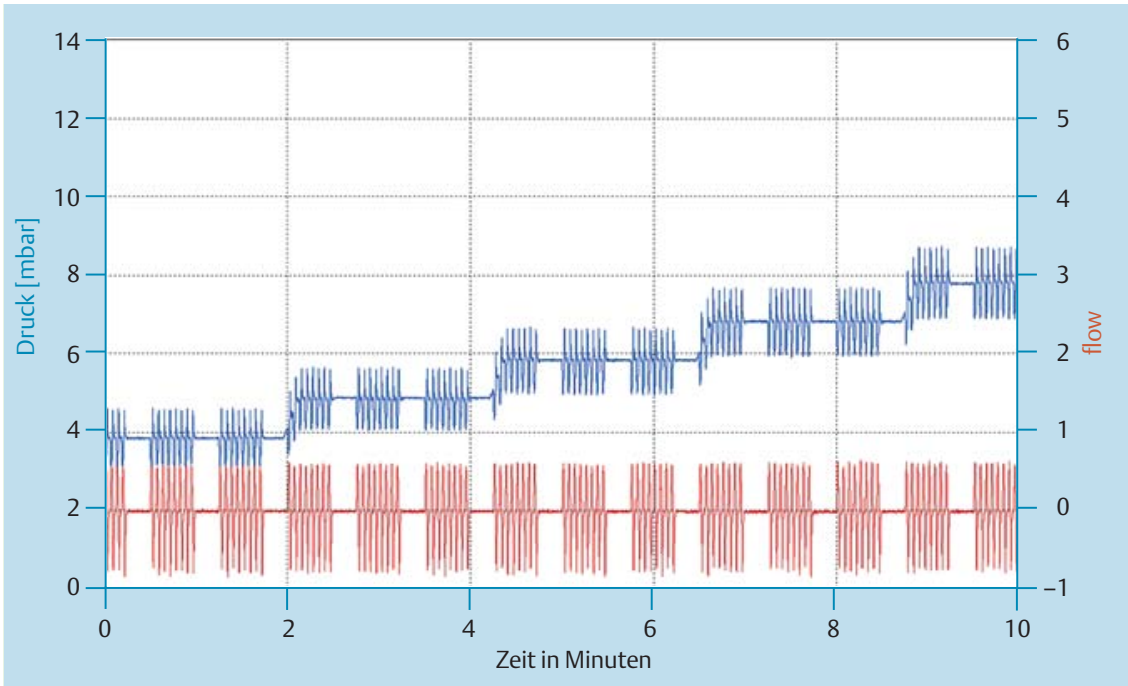

Abb.9 Open-Loop-Druck (oben) und -Fluss (unten) während repetitiver obstruktiver Apnoen. Registrierzeit 10 min. Verhalten von Gerät B: Ausgangsdruck 4 mbar Nach 3 Apnoen Druckanstieg jeweils 1 mbar, obere Druckgrenze 9 mbar.

Schritten einem oberen Druck von 10 mbar zu nähern. Ein Druck von 10 mbar wurde nach etwa 8-10 repetitiven Apnoen erreicht. ( $\bullet$ Abb. 8).

Gerät B: Dieses Gerät reagierte bei repetitiven Apnoen am Ende jeweils der 3. Apnoe mit 1 mbar Druckanstieg und zeigt damit im Gegensatz zu Gerät A eine lineare Druckanstiegs-Charakteristik. Ein Druck von 10 mbar wurde nach etwa 20 repetitiven Apnoen erreicht $(\bullet$ Abb. 9 ).

Gerät C: Hier fanden wir mit 2 mbar den höchsten Druckanstieg im Rahmen eines einzelnen Druckschritts. Dieses Gerät reagierte allerdings erst nach 6 Apnoen mit jeweils 2 mbar Druckanstieg. Nach 13 Apnoen wurde der Druck 10 mbar erreicht. ( Abb. 10).

\section{Testlauf 2}

Open loop, offene Apnoen: Bei diesem Test wurde dasselbe Protokoll wie in Testlauf 1 verwendet. Gerät A und B reagierten trotz fehlender Obstruktion mit einem im Vergleich zu Testlauf 1 identischen Druckanstieg ( $\bullet$ Abb. 11, 12). Gerät C differenzierte als einziges zwischen geschlossenen und offenen Apnoen (1) Abb. 13).

\section{Testlauf 3}

Closed loop: Die durch die Druckerhöhung reduzierte Obstruktion der oberen Luftwege (aus Apnoe wird Hypopnoe, aus Hypopnoe wird Flattening) führte bei den Geräten A, B und C zu unterschiedlichen Mustern der Druckanpassung.

Gerät A: Nach mehreren Apnoen stieg der Druck von 4 auf 6 mbar an. Unter den jetzt resultierenden Hypopnoen fiel der Druck langsam ab. Die unterhalb von 6 mbar erneut auftretenden Apnoen führten bei 5,5 mbar zu einem PAP-Anstieg auf 7 mbar, sodass erneut Hypopnoen generiert wurden. Danach wiederholte sich der Vorgang (siehe $\bullet$ Abb. 14).

Gerät B: Der Druckanstieg, ausgelöst durch die Apnoen, erfolgte bis 7,5 mbar. Die jetzt auftretenden Hypopnoen führten im folgenden Verlauf weder zu einem Druckanstieg noch zu einem Abfall (siehe $\bullet$ Abb. 15).

Gerät C: Die Apnoen führten zu einem Druckanstieg bis zu 9 mbar, sodass der Flowgenerator Hypopnoen simulierte. Nach etwa 10 Minuten fiel daraufhin der PAP langsam ab. Die daraufhin ausgelöste Apnoe ließ den Druck erneut um 1 mbar ansteigen (- Abb. 16). 


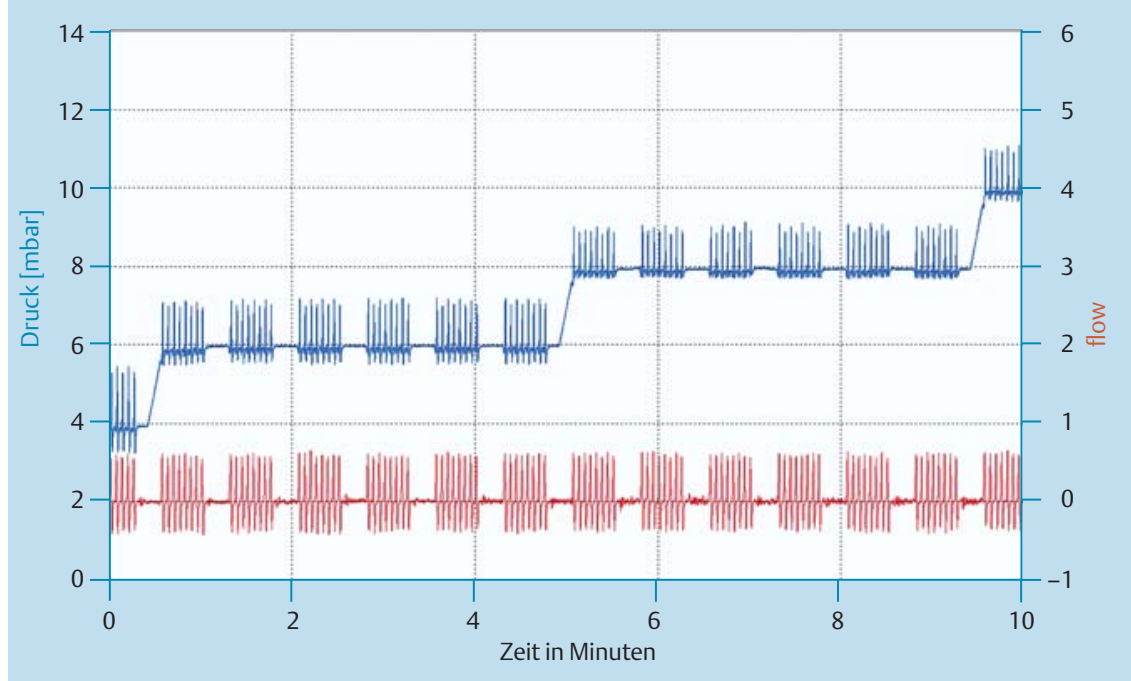

Abb. 10 Open-Loop-Druck (oben) und -Fluss (unten) während repetitiver obstruktiver Apnoen. Registrierzeit 10 min. Verhalten von Gerät C: Ausgangsdruck 4 mbar. Nach 6 Apnoen Druckanstieg jeweils 2 mbar, obere Druckgrenze 10 mbar.

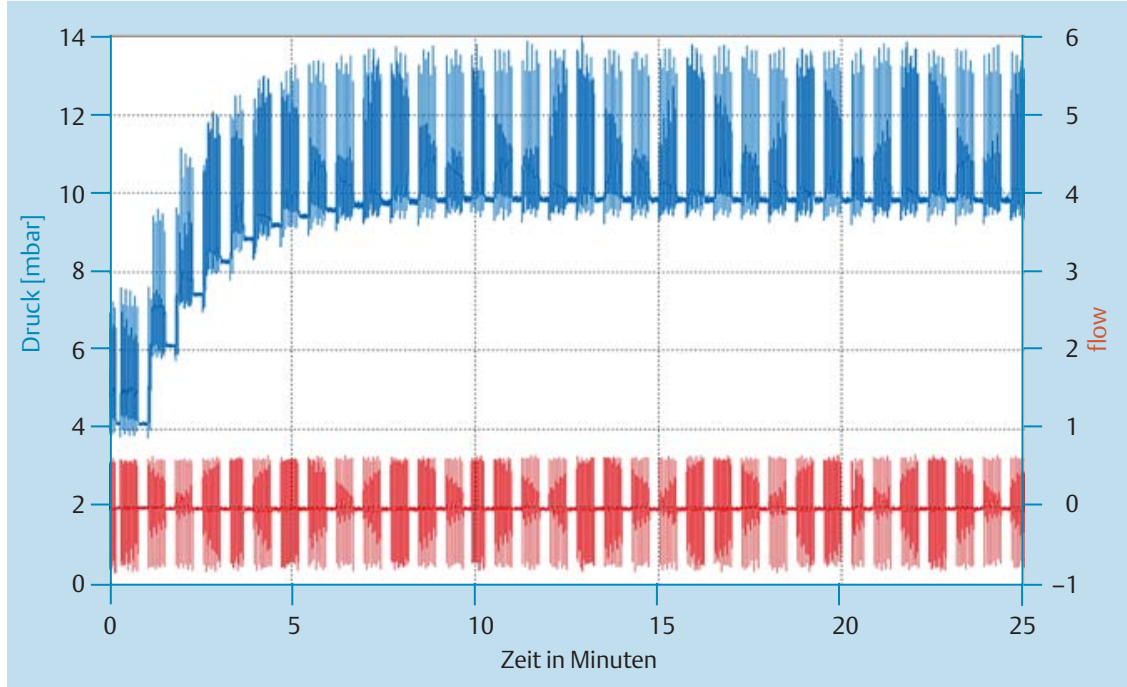

Abb. 11 Open-Loop-Druck (oben) und -Fluss (unten) während repetitiver offener Apnoen. Registrierzeit 25 min. Verhalten von Gerät A: Ausgangsdruck $4 \mathrm{mbar}$. Trotz offener repetitiver Apnoen Druckanstieg bis 10 mbar.

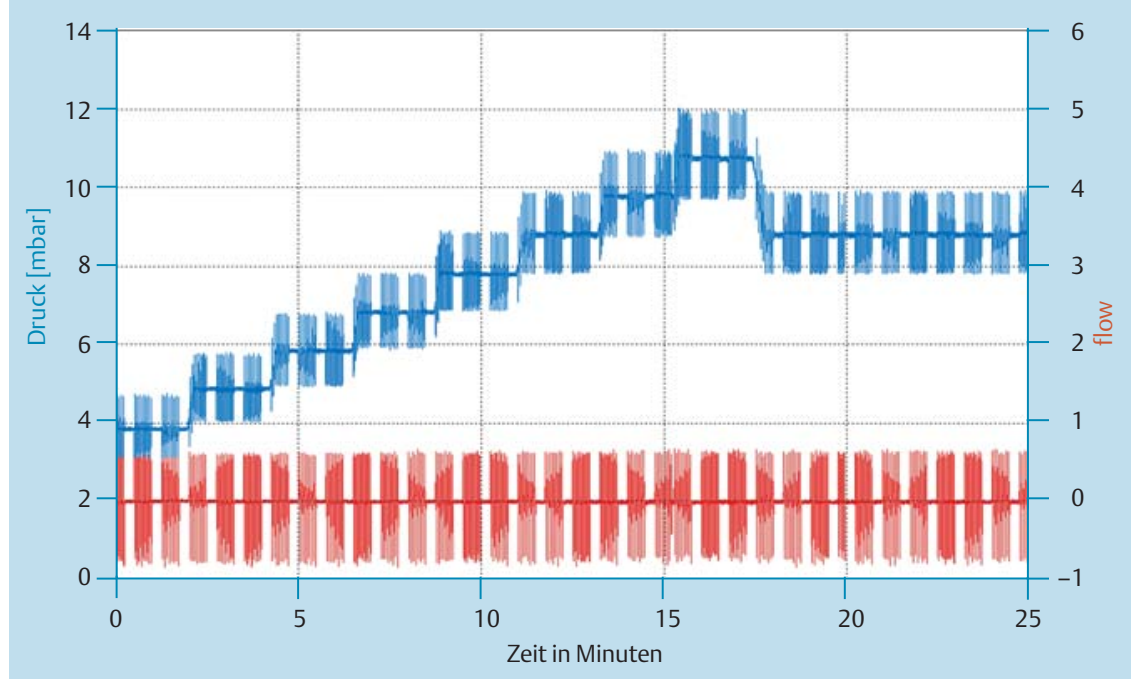

Abb. 12 Open-Loop-Druck (oben) und -Fluss (unten) während repetitiver offener Apnoen. Registrierzeit 25 min. Verhalten von Gerät B: Ausgangsdruck 4 mbar. Trotz offener repetitiver Apnoen Druckanstieg bis $11 \mathrm{mbar}$, danach 9 mbar. 


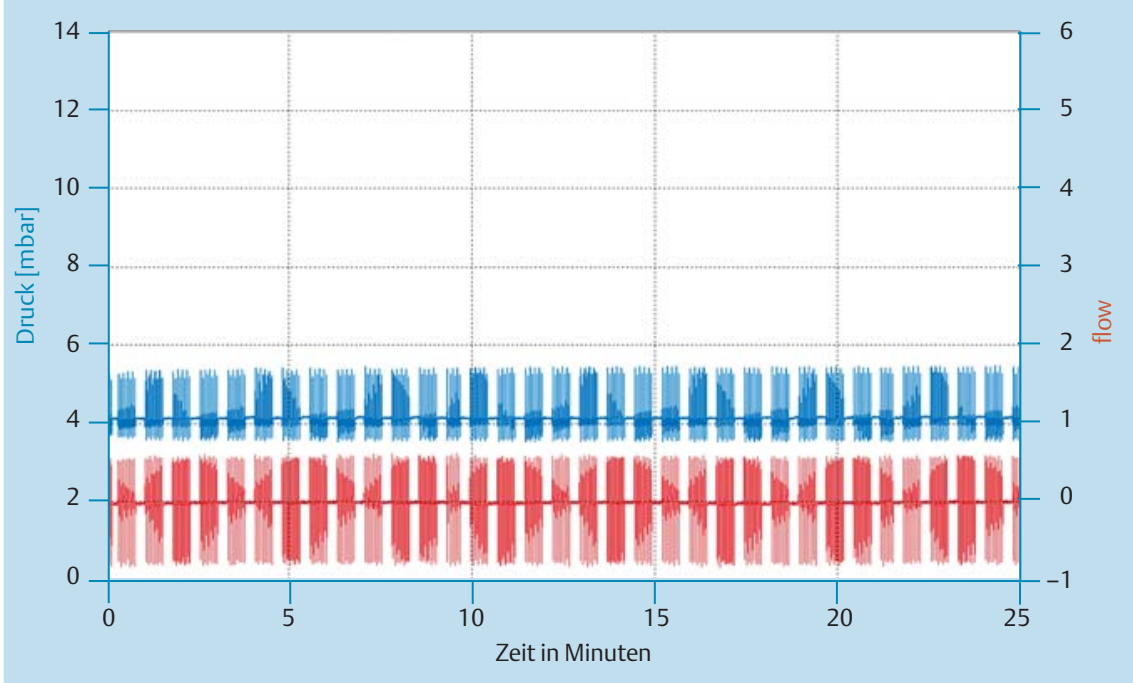

Abb. 13 Open-Loop-Druck (oben) und -Fluss (unten) während repetitiver offener Apnoen. Registrierzeit 25 Min. Verhalten von Gerät C: Ausgangsdruck 4 mbar. Offene repetitive Apnoen werden erkannt: richtige Reaktion, da kein Druckanstieg.

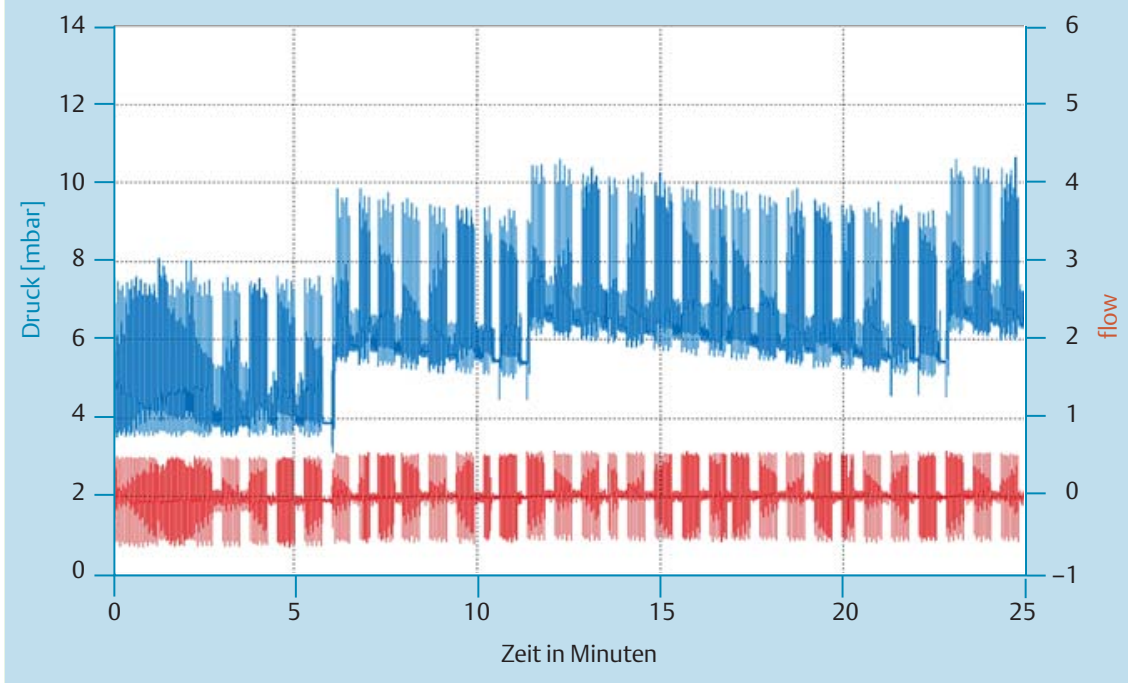

Abb. 14 Closed-Loop-Druck (oben) und -Fluss (unten) während repetitiver obstruktiver Apnoen. Registrierzeit $25 \mathrm{~min}$. Verhalten von Gerät A: Ausgangsdruck 4 mbar. Nach mehreren Apnoen Druckanstieg auf 6 mbar. Unter resultierenden Hypopnoen langsamer Druckabfall bis zum Kollapsdruck von 5,5 mbar und erneuter PAP-Anstieg auf 7 mbar (Näheres siehe Text).

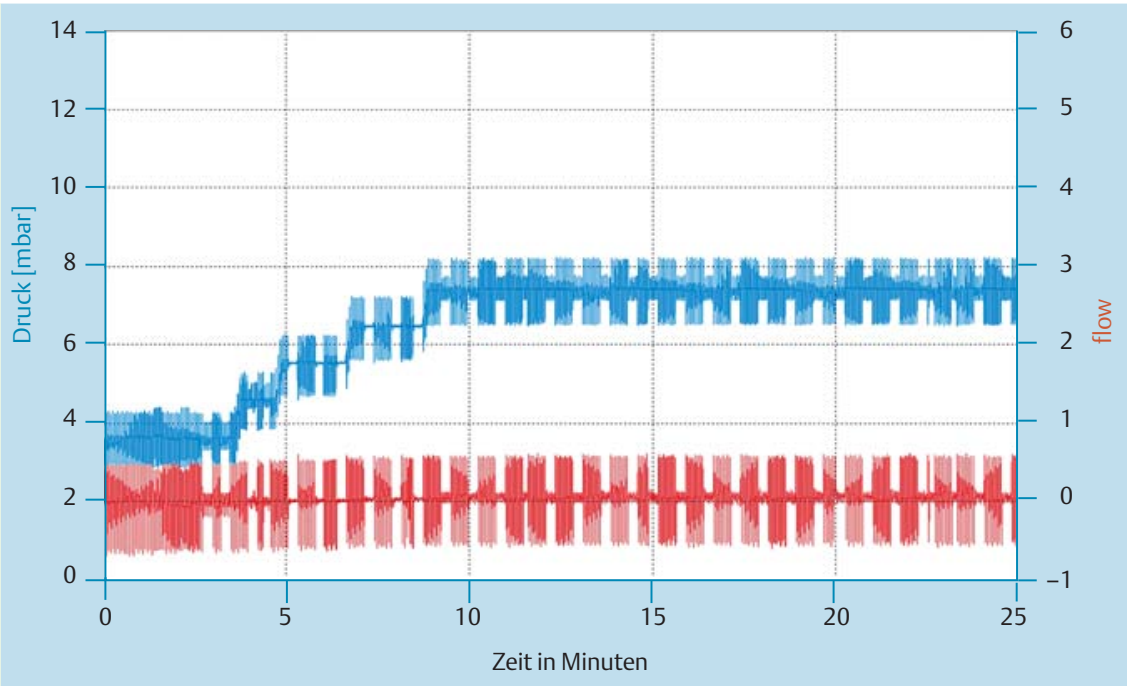

Abb. 15 Closed-Loop-Druck (oben) und -Fluss (unten) während repetitiver obstruktiver Apnoen. Registrierzeit 25 min. Verhalten von Gerät B: Ausgangsdruck 4 mbar. Druckanstieg 7,5 mbar. Bei Hypopnoen konstanter Druckverlauf bei 7 mbar (Näheres siehe Text). 


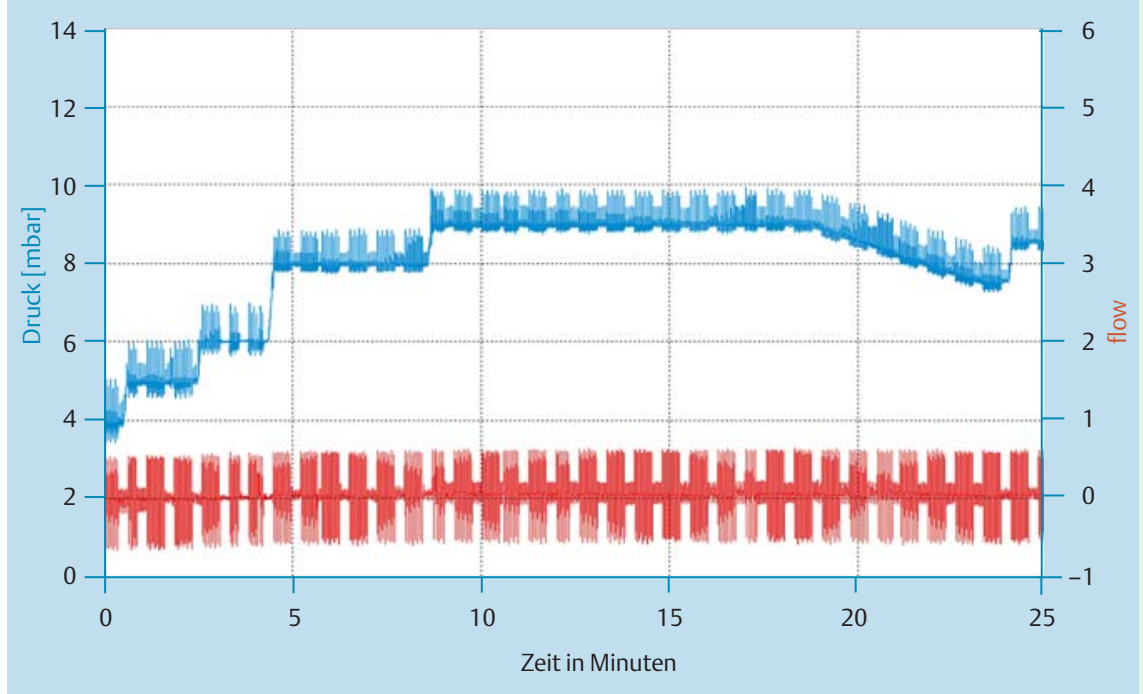

Abb. 16 Closed-Loop-Druck (oben) und -Fluss (unten) während repetitiver obstruktiver Apnoen. Registrierzeit 25 min. Verhalten von Gerät C: Ausgangsdruck 4 mbar. Druckanstieg bis Hypopnoen bei 9 mbar. Nach 10 min langsamer Druckabfall bis zum Kollapsdruck mit Apnoe: Druckanstieg um 1 mbar (Näheres siehe Text).

\section{Diskussion \\ $\nabla$}

Die von uns durchgeführte Testung gibt nur partielle Informationen über die Algorithmen der untersuchten Geräte wieder. Insbesondere untersuchten wir nicht den zusätzlichen Einfluss von Schnarchgeräuschen, die sehr leicht detektiert werden können $[10,11]$ und die im Steueralgorithmus einiger Geräte zu einer beschleunigten Druckerhöhung führen. Zum Beispiel wird in Gerät B der Druckanstieg bis 10 mbar bei leichten Hypopnoen von 9 min durch Schnarchen auf 5,5 min reduziert [5]. Außerdem kann nicht ausgeschlossen werden, dass weitere Signale (z. B. kardiale Druckoszillationen als Marker für offene Apnoen [12], die im Flussgenerator nicht berücksichtigt wurden, die Druckregulation beeinflussen. Diese und weitere Signale wie der obstructive pressure peak (OPP) [13] sollten in die Lungensimulatoren implementiert werden. Es muss deshalb betont werden, dass fehlende Reaktionen der APAP-Geräte auch durch nicht simulierte Phänomene bedingt sein könnten.

Aufgrund der hohen Sensitivität des Drucksensors und der zeitlichen Auflösung des Drucksignals (1000 Hz) erscheint zu Beginn der Exspiration ein hoher Peak, der durch die extrem geringe Compliance von Simulator und Dummy erklärt werden kann. Ähnliche Druckkurven findet man auch in der ersten Bench-Studie von Farré. Weitere Verbesserungen des Complianceverhaltens sind deshalb erforderlich.

Obwohl keine obere Druckbegrenzung vorgegeben war, pendelten sich alle 3 getesteten Geräte während des Testlaufs 1 trotz persistierender obstruktiver Apnoen bei einem Wert von etwa 10 mbar ein. Die Geschwindigkeit ist allerdings unterschiedlich. Zur Charakterisierung der Druckanstiegsdynamik kann die Zeit ermittelt werden, die es dauert, bis ausgehend von 4 mbar der Druck 10 mbar erreicht wird. Diese hängt von der Druckerhöhung, der Zeitdauer der jeweiligen Normalatmung und der Dauer der Apnoen ab. Die Zeitdauer einer Apnoe- und der folgenden Normalatmung wurde im Experiment von Rigau [5] mit jeweils 13 Sekunden programmiert. Durch die verschiedenen Algorithmen bedingt, dauerte es bei Gerät A 7 min, bei Gerät B $11 \mathrm{~min}$ und bei Gerät C $13 \mathrm{~min}$, bis 10 mbar CPAP erreicht wurden. Unsere Testung konnte bei gering differierender Länge der Apnoe und Normalatmung diese Ergebnisse bestätigen.
Wie in der Untersuchung von 2006 [5] wird durch die Geräte A und $\mathrm{B}$ weiterhin nicht zwischen offenen und geschlossenen Apnoen unterschieden.

Damit wurde durch die Hersteller innerhalb eines Zeitraums von 2 Jahren keine Änderung der Gerätekonfiguration bzgl. dieser 2 Algorithmen vorgenommen.

Sehr viel komplexer fiel die Testung bei der Überprüfung des Rückkopplungsverhaltens aus. Überraschend war, dass bei Hypopnoen kein weiterer Druckanstieg, sondern Druckabsenkung, Druckkonstanz oder Druckkonstanz mit späterer Druckabsenkung resultierte. Dieses Verhalten weist darauf hin, dass die Algorithmen der Geräte nur bei länger persistierenden Apnoen höhere Druckniveaus ansteuern.

Um das Druckverhalten dieser mit aufwendigen Programmen ausgestatteten Geräte noch genauer charakterisieren zu können, sind deshalb Messungen über mehrere Stunden erforderlich, da das Langzeitverhalten von akuten Messungen (30-60 min Dauer) deutlich differieren dürfte.

Bedeutung von Bench-Tests: In der klinischen Situation kann die Reaktion von APAP-Geräten auf eine Atemstörung, bedingt durch den variablen Input, schlecht interpretiert werden. Im Einzelfall kann durch Messungen im Schlaflabor das Regelverhalten des APAP-Gerätes in der betreffenden Nacht bei dem individuellen Patienten abgeschätzt werden. Im häuslichen Bereich ist dies jedoch kaum möglich und Hinweise zur Behandlungsqualität können nur den Gerätespeichern entnommen werden. Insbesondere durch die zunehmend eingeschränkten Möglichkeiten zu Schlaflabor-Kontrolluntersuchungen sind daher Informationen zum Regelverhalten und zur diagnostischen Sicherheit bezüglich des Auftretens von zentralen Apnoen sehr wichtig. Im Hinblick auf die unterschiedlichen Algorithmen der APAP-Geräte sollte deshalb die Ersteinstellung mit einem APAP-Gerät polysomnographisch überprüft werden.

Die Bench-Untersuchungen weisen darauf hin, dass durch definierte Vorgaben von Flusskurven und Simulation der pharyngealen Obstruktion die Reaktion exakt beurteilt werden kann. Allerdings existieren unterschiedliche Testverfahren, sodass eine Standardisierung wünschenswert wäre. Vorgeschlagen wird z. B. ein Prüfprotokoll über 60 bzw. 83 Minuten, bei dem verschiedene Obstruktionsgrade, zentrale Apnoen, Schnarchen und Leckage simuliert werden [14]. Aus den Ergebnissen werden 5 dimensionslose Kennzahlen definiert, die u.a. die Dynamik beim Eintreten 
respiratorischer Ereignisse betreffen. Als geeignete Plattform zur Erstellung von Beurteilungskriterien könnte eine gemeinsame Arbeitsgruppe der mit diesem Thema befassten wissenschaftlichen Arbeitsgruppen dienen [15].

Therapeutische APAP- als auch speziell für die Titration entwickelte Geräte werden häufig aus Kosten- und Zeitgründen zur automatischen Titration mit Ermittlung des fixen CPAP eingesetzt [16-19]. Die Bestimmung der 95\% Percentile, d.h. der Druck, der nur in $5 \%$ der Zeit überschritten werden darf, hängt entscheidend von der Druckanstiegsdynamik ab. Die genaue Kenntnis des Titrations-Algorithmus ist deshalb für die Druckempfehlung des häuslichen CPAP besonders hilfreich.

\section{Schlussfolgerungen \\ $\nabla$}

Durch Bench-Tests kann der Algorithmus der Auto-CPAP-Geräte regelmäßig überprüft und Veränderungen der Software erkannt werden. Die Geräte zeigen deutliche Unterschiede in der Geschwindigkeit des Druckanstiegs. Durch die Kenntnis der verschiedenen Reaktionen ist ein individualisierter und gezielterer Geräteinsatz möglich. Es ist deshalb denkbar, dass durch Kenntnis und eventuell Verbesserung des Druckverhaltens der AutoCPAP-Geräte und Berücksichtigung der Obstruktionsdynamik der oberen Atemwege die Therapie weiter optimiert werden kann.

\section{Interessenkonflikte}

$\nabla$

K. H. Rühle und G. Nilius erhielten finanzielle Unterstützung wissenschaftlicher Projekte von Weinmann, Respironics, Resmed, Heinen und Löwenstein, Fisher und Paykel. Der Artikel wurde finanziell nicht unterstützt.

\section{Literatur}

1 Rühle KH, Raschke F, Fietze I et al. Titration und Therapie mittels Positiv-Druckatmung bei schlafbezogenen Atemstörungen (SBAS). Somnologie 2004; 8: 95 - 109

2 Farré R, Montserrat JM, Rigau J et al. Response of automatic continuous positive airway pressure devices to different sleep breathing patterns: a bench study. Am J Respir Crit Care Med 2002; 166: 469- 473

3 Abdenbi F, Chambille B, Escourrou P. Bench testing of auto-adjusting positive airway pressure devices. Eur Respir J 2004; 24: 649-658

4 Lofaso F, Desmarais G, Leroux K et al. Bench evaluation of flow limitation detection by auto-CPAP devices. Chest 2006; 130: $343-349$

5 Rigau J, Montserrat JM, Wöhrle H et al. Bench model to simulate upper airway obstruction for analyzing automatic CPAP devices. Chest 2006; 130: $350-361$

6 Randerath WJ, Schraeder O, Galetke W et al. Autoadjusting CPAP therapy based on impedance efficacy, compliance and acceptance. Am J Respir Crit Care Med 2001; 163: 652 - 657

7 Massie CA, McArdle N, Hart RW et al. Comparison between automatic and fixed positive airway pressure therapy in the home. Am J Respir Crit Care Med 2003; 167: 20-23

8 Hukins C. Comparative study of autotitrating and fixed-pressure CPAP in the home: a randomized, single-blind crossover trial. Sleep 2004; 27: $1512-1523$

9 Ayas NT, Patel SR, Malhotra A et al. Auto-titrating versus standard continuous positive airway pressure for the treatment of obstructive sleep apnea: results of a meta-analysis. Sleep 2004; 27: 249-253

10 Dalmasso F, Prota $R$. Snoring: analysis, measurement, clinical implications and applications. Eur Respir J 1996; 9: 146-159

11 Lofaso F, Leroux K, Quera-Salva MA et al. Snoring detection during autonasal continuous positive airway pressure. Eur Respir J 2002; 19: 108 112

12 Ayappa I, Norman RG, Rapoport DM. Cardiogenic oscillations on the airflow signal during continuous positive airway pressure as a marker of central apnea. Chest 1999; 116: 660-666

13 Rühle KH, Domanski U, Schwaibold $M$ et al. Obstructive Pressure Peak zur Differenzierung von obstruktiven und zentralen Apnoen bei der Therapie mit Auto-CPAP. Somnologie 2008; 12 (Suppl): 29

14 Netzel T, Birkenseer M, Grohmann U et al. Aktiver Lungen-und Obstruktionssimulator zur Bewertung von Beatmungsgeräten. Somnologie 2008; 12 (Suppl): 28

15 Brown LK. Autotitrating CPAP: how shall we judge safety and efficacy of a „black box“? Chest 2006; 130: 312-314

16 Teschler H, Berthon-Jones M, Thompson AB et al. Automated continuous positive airway pressure titration for obstructive sleep apnea syndrome. Am J Respir Crit Care Med 1996; 154: 734-740

17 Lloberes P, Ballester E, Montserrat JM et al. Comparison of manual and automatic CPAP titration in patients with sleep apnea/hypopnea syndrome. Am J Respir Crit Care Med 1996; 154: 1755 - 1758

18 Stradling JR, Barbour C, Pitson DJ et al. Automatic nasal continuous positive airway pressure titration in the laboratory: patient outcomes. Thorax 1997; 52: 72 - 75

19 Masa JF, Jimenez A, Duran J et al. Alternative methods of titrating continuous positive airway pressure: a large multicenter study. Am J Respir Crit Care Med 2004; 170: 1218 - 1224 